\title{
La clase en la educación superior, forma organizativa esencial en el proceso de enseñanza-aprendizaje
}

\section{Rafael Carlos Hernández-Infante}

orcid.org/00oo-0002-6555-5459

Universidad Regional Autónoma de los

Andes (Ecuador)

rafaelcarlos_docente@outlook.com

\section{María Elena Infante-Miranda}

orcid.org/0000-0002-0828-1383

Universidad Regional Autónoma de los

Andes (Ecuador)

m_infante_docente@hotmail.com

\section{Resumen}

El proceso de enseñanza-aprendizaje en la educación superior tiene varias formas organizativas; independientemente de la importancia que adquiere cada una para el desarrollo de los saberes concebidos en la malla curricular, de las competencias necesarias para determinada profesión, se considera que la clase constituye una de las formas fundamentales de organización de dicho proceso. En la clase, debe lograrse la unidad entre lo instructivo y lo educativo, principio fundamental a tener en cuenta en todo el proceso. El objetivo de este artículo es analizar consideraciones sobre la clase en la educación superior, como forma organizativa esencial en el proceso de enseñanza-aprendizaje, a partir de las características de sus diferentes tipos. En su elaboración, se emplean métodos de investigación del nivel teórico que permiten el procesamiento de la información, la caracterización del objeto de investigación, determinar sus fundamentos teóricos y metodológicos. Como conclusión, se plantea que la clase en la educación superior puede adoptar diferentes tipologías: conferencia, clase práctica, seminario y la clase encuentro, fundamentalmente; cada una tiene características peculiares que permiten tratar conocimientos, desarrollar habilidades y valores, con la participación activa de los estudiantes, lo que favorece su formación integral, meta suprema del sistema educativo.

\section{Palabras clave}

Enseñanza; formación; educación; instrucción; proceso de enseñanza; método de enseñanza (Fuente: Tesauro de la Unesco).

Recepción: 2015-10-22 | Envío a pares: 2016-07-26 | Aceptación por pares: 2016-08-13 | Aprobación: 2016-08-30 


\title{
The Class in Higher Education: An Essential Organizational Form in the Teaching-learning Process
}

\begin{abstract}
The teaching-learning process in higher education has several organizational forms. Irrespective of the importance each one acquires to develop the knowledge envisaged in the curriculum, or the skills and competence necessary for a given profession, the class is considered to be one of the fundamental forms of organization in that process. Unity between what is instructive and what is educational must be achieved in the class; this is a fundamental principle to bear in mind throughout the process. The aim of this article is to analyze considerations about the class in higher education, as an essential organizational form in the teaching-learning process, beginning with the characteristics of the different types of classes. Theoretical research methods are used to process the information, characterize the object of the study, and determine its theoretical and methodological basis. It is argued, in conclusion, that the class in higher education can adopt different arrangements: a conference, a practical class, a seminar or a class encounter. Each one has particular features that allow knowledge to be addressed and skills and values to be developed, with the active participation of the students. This favors their comprehensive preparation, which is the ultimate goal of the educational system.
\end{abstract}

\section{Keywords}

Teaching; Training; Education; instruction; teaching process; teaching method (Source: Unesco Thesaurus). 


\section{A aula no ensino superior: forma organizativa essencial no processo de ensino-aprendizagem}

\section{Resumo}

O processo de ensino-aprendizagem no ensino superior tem várias formas organizativas; independentemente da importância que cada uma adquire para o desenvolvimento dos saberes concebidos na grade curricular, das competências necessárias para determinada profissão, considera-se que a aula constitui uma das formas fundamentais de organização desse processo. Na aula, deve-se conseguir a unidade entre o instrutivo e o educativo, principio fundamental a ser considerado em todo o processo. O objetivo deste artigo é analisar considerações sobre a aula no ensino superior, como forma organizativa essencial no processo de ensino-aprendizagem, a partir das características de seus diferentes tipos. Em sua elaboração, empregam-se métodos de pesquisa do nível teórico que permitem o processamento da informação, a caracterização do objeto de pesquisa bem como a determinação de seus fundamentos teóricos e metodológicos. Como conclusão, propõe-se que a aula no ensino superior pode adotar diferentes tipologias: conferência, aula prática, seminário e a aula encontro, fundamentalmente; cada uma tem características peculiares que permitem tratar conhecimentos, desenvolver habilidades e valores, com a participação ativa dos estudantes, o que favorece sua formação integral, principal meta do sistema educativo.

\section{Palavras-chave}

Educação; ensino; formação; instrução; método de ensino; processo de ensino (Fonte: Tesauro da Unesco). 


\section{Introducción}

La universidad, en la actualidad, tiene como una de sus más sustanciales metas la de responder a los retos que le sitúa el complejo contexto contemporáneo. Su propósito esencial es lograr la formación integral de los futuros profesionales, los que aportarán con su labor al desarrollo de la sociedad (Tünnermann, 2006; Tobón, 2010).

En el ámbito universitario se desarrollan diferentes procesos que contribuyen a ese propósito (MES, 2006): docencia, investigación y extensión universitaria (Guzmán, 2014) o vinculación con la sociedad (Araque, 2012), como también se le denomina, estrechamente relacionados. El proceso docente, dada su naturaleza, tiene la particularidad de integrarlos a todos; por ello existe una preocupación permanente en la comunidad educativa en cuanto a su necesario perfeccionamiento.

El proceso docente presenta diferentes formas organizativas; se consideran como las principales: la clase, la práctica de estudio, la práctica laboral, el trabajo investigativo, la autopreparación de los estudiantes y la consulta (MES, 1991). Independientemente de la importancia de cada una para el desarrollo de las materias que conforman el plan de estudio o la malla curricular en determinada carrera, se considera la clase como la forma fundamental para su organización (Bermúdez y Rodríguez, 1996).

La clase, entendida como el contexto donde se produce la interacción del docente y de los educandos, y de estos entre sí — sea o no de manera formal en un aula - con el propósito de dar cumplimiento a objetivos trazados (Hernández e Infante, 2011), posibilita la formación integral de los estudiantes. El proceso de enseñanza-aprendizaje se materializa, con toda su riqueza y diversidad, en su desarrollo, en cualquier sistema educativo.

En el nivel universitario se requiere que la clase evidencie la creatividad del docente para motivar a los estudiantes por el estudio, la ampliación de sus conocimientos, la participación activa en su adquisición y el establecimiento de sus nexos con la futura profesión. Las razones expuestas hacen que en el presente trabajo se tome como centro de atención esta forma de organización del proceso, teniendo consciencia de la necesidad de su mejora constante.

Aunque se reconoce la complejidad de la clase, la que debe responder a las exigencias de la carrera, de la materia y, a la vez, a las características de los estudiantes, ante las expectativas que genera en cuanto a su contribución a la formación integral de estos, en resumen, su importancia en el desarrollo del proceso de enseñanza-aprendizaje, la práctica pedagógica revela limitaciones en su concepción didáctica por lo que se realiza una investigación al respecto. Los resultados obtenidos se presentan en este artículo, el que tiene como objetivo socializar, en la comunidad educativa, las recomendaciones metodológicas elaboradas para contribuir al adecuado desarrollo de la clase, forma organizativa esencial en el proceso de enseñanza-aprendizaje en el nivel superior.

\section{Metodología}

Se emplean métodos de investigación del nivel teórico: análisis-síntesis, inducción-deducción e histórico-lógico. Estos permiten el procesamiento de la información, la caracterización del objeto de investigación, la determinación de sus fundamentos teórico-metodológicos, así como la elaboración de conclusiones a partir del objetivo trazado.

Se utilizan métodos del nivel empírico para estudiar la realidad escolar: la observación y la entrevista. La observación del proceso de enseñanza-aprendizaje adquiere un carácter estructurado, al tener como base una guía en la que se establecen los indicadores que se deberán tener en cuenta; es sistemática, pues abarca un semestre, y de campo, al realizarse en el contexto natural del proceso. Se seleccionan intencionalmente docentes para ser entrevistados. 


\section{La clase en la enseñanza superior}

El proceso de enseñanza-aprendizaje en la enseñanza superior debe concebirse en función de la formación de los estudiantes como futuros profesionales. Desde la clase, estos han de prepararse para que sean capaces de identificar problemas relacionados con su carrera, y, por consiguiente, proponer por soluciones a los mismos. Este proceder permite dirigir la docencia universitaria con un enfoque profesional (Didriksson, 2003), en función del desarrollo de modos de actuación en el alumnado.

Para dar cumplimiento a ese cometido, la planificación de una clase debe contemplar actividades investigativas para desarrollar por los estudiantes. Las mismas permiten el empleo de variados métodos de investigación que contribuyen al desarrollo de su independencia cognoscitiva, gradualmente, dando paso a la autorregulación, a la autonomía (Castellanos et al., 2002), aspectos esenciales para lograr como parte de su formación profesional y de la educación de su personalidad.

Es necesario que el docente conciba su clase de manera tal que permita a los educandos desempeñar un papel activo en la construcción de los conocimientos, en el desarrollo de habilidades y valores, en dependencia de las características de la materia, del nivel que cursen y de sus individualidades. Debe estimular un aprendizaje participativo (Mestre et al., 2008), en el cual facilite la actuación de los educandos, estimulando su creatividad y accionar consciente, lo que repercute en su formación integral.

El adecuado desarrollo de una clase requiere que en la misma se trate el contenido de enseñanza-aprendizaje con rigor científico (Argudín, 2000). Para ello deben emplearse fuentes actualizadas y suficientes, las que han de permitir al docente realizar una orientación precisa a los estudiantes para que puedan profundizar en lo tratado mediante la utilización de métodos de investigación.

En la enseñanza superior la clase puede adoptar diferentes formas. El docente planifica el tipo de clase que va a desarrollar a partir de los objetivos que deben ser cumplidos en el programa que imparte; tiene en cuenta, además, la modalidad de estudios, las características del contenido de enseñanza-aprendizaje, así como las particularidades del grupo de estudiantes a los que va dirigida, entre otros elementos.

El análisis de un programa puede indicar que resulta necesaria la presentación de fundamentos teóricos referidos a un tema, los que por su complejidad y amplitud deben ser expuestos en una sesión de clases, posibilitando a los alumnos su aplicación en momentos posteriores. En la etapa de preparación o planificación del trabajo docente por ejecutar, se decide el tipo de clase que resulta más conveniente para lograr el objetivo propuesto, desarrollar el contenido de enseñanza-aprendizaje, utilizando los métodos más adecuados.

En este caso, el docente debe planificar una clase centrada en su exposición (clase teórica o conferencia), con el fin de abarcar los fundamentos esenciales que deben ser tratados, sin descuidar la participación de los estudiantes. Esta clase ha de contar con medios o recursos didácticos (Cabero, 2001) que posibiliten la transmisión del contenido de enseñanza-aprendizaje y sirvan de apoyo a los métodos seleccionados.

En el proceso de preparación de las clases correspondientes al ejemplo que se presenta, el docente debe concebir una o varias, según se requiera, para propiciar la aplicación de los aspectos teóricos estudiados (clase práctica). Este tipo de clase posibilita el desarrollo de las habilidades, las que son propias de la materia y las necesarias para la futura profesión, concebidas en el modelo del profesional o perfil de egreso de la carrera, en el programa de la disciplina y en el de la asignatura. Las decisiones metodológicas que realiza el docente revisten importancia, en tanto permiten que el proceso de enseñanza-aprendizaje se desarrolle adecuadamente.

En la preparación de cada clase se debe tener en cuenta, además, el resultado del diagnóstico que 
se realice. Este es un proceso de carácter permanente, sistemático e integrador (Zilberstein y Silvestre, 2000), que informa al profesor sobre el estado actual del proceso, lo que le permite ofrecer un tratamiento pedagógico diferenciado para atenuar las debilidades detectadas y estimular las potencialidades en cada uno de los educandos.

La didáctica de la enseñanza superior (Escudero, 2006; Rué, 2007; Fuentes, 2009) le proporciona al docente diferentes tipos de clases o formas de docencia, las que deben ser planificadas teniendo en cuenta los elementos ya mencionados. Dentro de la amplia gama de maneras para referirse a los tipos de clase que pueden desarrollarse en este nivel, a continuación se mencionan los que se consideran de mayor interés, atendiendo al objetivo trazado.

Álvarez de Zayas (1999) cataloga la clase en la enseñanza superior como: conferencia, clase práctica, seminario, concepción asumida por el MES (2007). Zabalza (2004) concibe clases teóricas, debates, trabajos (individuales o en grupo), prácticas (de aula o laboratorio) y tutoría. La Universidad del País Vasco (2006) habla de clases magistrales, teóricas, seminarios, prácticas de aula, de laboratorio, de ordenador, clínicas, de campo, deportivas y talleres (industriales o no). En criterio de Borgobello, Peralta y Roselli (2010) las clases se clasifican en teóricas y prácticas.

Independientemente de la utilidad de estos fundamentos, en la presente investigación se toma la clasificación más general, la que concibe clases teóricas (conferencias) y clases prácticas, dentro de las que se encuentra una variada tipología, como se menciona. Esta decisión obedece a que de esta forma se hace una distinción precisa entre clases dirigidas a tratar presupuestos teóricos por parte del docente y otras encaminadas al desarrollo de las habilidades en los alumnos.

Los referentes teóricos analizados sustentan la realización del diagnóstico al proceso de enseñanzaaprendizaje en la Universidad Regional Autónoma de los Andes, en Ecuador, con el propósito de profundizar en la situación detectada, referida a limitaciones en la concepción didáctica de la clase, como forma organizativa esencial del mismo. De manera intencional se selecciona la carrera de Derecho, pues la misma se prepara para su acreditación, proceso que exige el logro de la excelencia académica.

La observación del proceso se organiza a partir de indicadores establecidos en una guía (tratamiento del contenido: rol del docente y del estudiante; estructura metodológica de la clase; orientación de tareas investigativas, comunicación de los actores que intervienen en el proceso, formas de evaluación). La observación se realiza de manera sistemática, pues abarca el periodo lectivo de octubre de 2014 a marzo de 2015; es de campo, pues se realiza en el contexto natural del proceso. Se observan tres clases de cada una de las materias que conforman la malla curricular de la carrera en cada uno de los tres grupos que la conforman.

Los principales resultados obtenidos mediante la observación se sintetizan a continuación. Se revelan potencialidades en el desarrollo de las clases observadas en cuanto a la comunicación que se logra entre docentes y estudiantes, y de estos entre sí; se crea un clima que favorece el desarrollo del proceso. Se aprecian dificultades relacionadas con otros indicadores, las que se analizan a continuación.

El tratamiento del contenido recae fundamentalmente en el docente, lo que limita la participación del estudiante en su adquisición. Cuando se orientan tareas investigativas, no se precisan objetivos, fuentes esenciales para la consulta, indicadores para la evaluación y maneras de presentación de los resultados. Predomina la heteroevaluación, en detrimento de otras formas que pueden ser utilizadas. La estructura metodológica que se sigue no difiere, aunque los objetivos por lograr sean diferentes.

A fin de continuar profundizando en esta situación se seleccionan informantes claves, inten- 
cionalmente, para ser entrevistados. Se escogen a partir de su experiencia de trabajo en la carrera (cinco años o más) y de los resultados obtenidos en su superación (poseer título de cuarto nivel: doctor o máster). Estos criterios llevan a determinar una muestra de cinco profesores, reconocidos en la institución por su labor docente. Las entrevistas son de tipo no estandarizada, predomina su carácter abierto, se ofrecen temáticas en correspondencia con el objetivo propuesto, para ser desarrolladas.

Los docentes expresan sus criterios sobre elementos para tener en cuenta en la preparación de la clase: planificación de sus intervenciones, participación de los estudiantes, orientación de tareas investigativas, formas de evaluación, comunicación con los alumnos; y de estos entre sí: concepción de la estructura metodológica de la clase, en correspondencia con los objetivos por lograr. Reconocen que privilegian el tratamiento del contenido por su parte, pues de esa manera abarcan lo que consideran se necesita tratar.

Expresan que la preparación de la clase se realiza de manera general, pero que en el salón, donde fluye la misma, son determinantes: el dominio que poseen de los temas, su experiencia en la docencia y en la profesión. La utilización de los métodos mencionados permite que el diagnóstico arroje una percepción directa sobre el estado que presenta el desarrollo de la clase en esta institución, a partir de la muestra seleccionada. La profundización en este aspecto es esencial por la importancia de esta forma de docencia en el desarrollo del proceso de enseñanza-aprendizaje.

El análisis de los resultados obtenidos mediante el diagnóstico revela insuficiencias en la concepción didáctica de la clase, en su conducción, lo que dificulta la participación de los estudiantes, y, por consiguiente, que no se aprovechen todas sus potencialidades para contribuir a su formación integral. Atendiendo a esta situación, la investigación realiza una propuesta de solución a la misma. Se elaboran recomendaciones metodológicas dirigidas a los docentes con el propósito de contribuir al adecuado desarrollo de la clase, forma organizativa esencial en el proceso de enseñanza-aprendizaje en la enseñanza superior.

\section{Recomendaciones metodológicas para el desarrollo de clases teóricas (conferencias)}

Es el tipo de clase que se caracteriza por la realización de una exposición sobre determinado tema, por parte del docente. Esta debe ser clara, precisa, actualizada y lógicamente estructurada; debe abarcar elementos esenciales, haciendo énfasis en los aspectos que resulten más complejos para los estudiantes. Es una clase idónea para explicar los fundamentos teóricos de un tema en cuestión, en ella se debe suscitar el análisis de los educandos, su reflexión sobre el tema, es decir, su participación activa. Deben emplearse métodos y medios de enseñanza-aprendizaje que despierten su interés para que profundicen en el mismo de manera independiente.

El adecuado desarrollo de una clase teórica requiere cumplir con su estructura metodológica (introducción, desarrollo y conclusiones), lo que incide favorablemente en el logro de sus objetivos. En la introducción se debe vincular el tema que se va a tratar con los ya estudiados, proceder que ayuda a la sistematización del contenido de enseñanza-aprendizaje de la materia (relaciones intramateria) (Fiallo, 1997). Resulta importante que se realice la comprobación de los conocimientos adquiridos por los estudiantes, evaluación que permite la retroalimentación necesaria para el desarrollo del proceso.

En esta parte de la clase el docente identifica el tema que se va a tratar, y da muestras de su maestría pedagógica al estimular su estudio por parte de los estudiantes; aunque la motivación no se restringe a un momento específico de la clase, todo lo contrario, debe mantenerse a lo largo de la misma. Un elemento significativo dentro de la introducción es la orientación hacia el objetivo planteado. 
Esta función didáctica (Valdés, 2013) permite que los educandos sepan lo que deben lograr en la clase, los aprendizajes esperados (Frade, 2014); es necesario puntualizar que dicha función debe estar presente en toda la clase.

En la introducción, el docente, además, debe realizar un análisis crítico de las fuentes bibliográficas que se deberán consultar. Es necesario que destaque las que son básicas para profundizar en el tema y las complementarias, las que están al alcance de los estudiantes, el enfoque del tema que aporta cada una, en resumen, que prepare las condiciones necesarias para que posteriormente los alumnos localicen dichas fuentes y las utilicen en su estudio independiente, que les permitirá ampliar y consolidar los conocimientos que exige el tema.

El desarrollo de una clase teórica es aquella parte en la que el docente realiza una exposición lógica, y científicamente fundamentada, del contenido de enseñanza-aprendizaje. El empleo adecuado de métodos de carácter participativo (González, 1990) o los llamados métodos activos (Ventosa, 2004) promueve el análisis y la reflexión en los alumnos; contribuye a que estos desempeñen un papel activo en la adquisición de los conocimientos.

El uso de medios de enseñanza-aprendizaje (Área, 2002; Bravo, 2003) incide favorablemente en el cumplimiento de los objetivos establecidos. Estos son de diferentes tipos: la palabra del docente, el pizarrón (donde se realizan importantes apuntes y esquemas sobre el contenido que se analiza), así como otros de carácter técnico que exigen la aplicación de las tecnologías de la información y las comunicaciones (TIC), según Aguilar (2003). Su empleo adecuado hace más vívido el aprendizaje de los estudiantes, lo facilita y estimula.

El desarrollo es la parte más extensa de este tipo de clase, incluye un considerable volumen de conocimientos. Por ello es necesario que el docente realice resúmenes parciales cada vez que lo consi- dere pertinente. Estos permiten a los estudiantes precisar los aspectos que se tratan, tomar notas de lo esencial y analizar las relaciones entre los diferentes elementos que se presentan. El docente, además, debe ir planteando interrogantes que le permitan conocer si los alumnos siguen la lógica de su exposición, si comprenden los fundamentos teóricos que se analizan, con el propósito de esclarecer lo que se requiera.

En este tipo de clase es necesario que el docente oriente y, posteriormente, controle correctamente el trabajo independiente de los estudiantes. Estos deben saber con precisión: qué deben estudiar, con qué propósito, qué fuentes fundamentales deben consultar, cómo y cuándo deben presentar sus resultados y cómo serán evaluados.

El momento para realizar dicha orientación debe decidirlo el profesor, atendiendo a la planificación que realiza de la clase. Puede hacerlo al concluir la misma o en algún momento que resulte propicio en su desarrollo. Lo esencial es que lo tratado en el aula sea profundizado por los alumnos mediante tareas docentes coherentemente elaboradas, que serán objeto de análisis en otros momentos del proceso.

Las conclusiones de la clase teórica presentan generalizaciones sobre el tema tratado. En esta parte de la clase el docente sintetiza los aspectos fundamentales, en correspondencia con los objetivos propuestos. Hace énfasis en lo que aporta lo estudiado al futuro desempeño profesional de los estudiantes. Realiza interrogantes que le permiten obtener información sobre la asimilación de los conocimientos expuestos; en consecuencia, precisa lo que se requiera, para ello puede emplear organizadores gráficos (Ontoria et al., 2006) que ayuden a resumir las ideas presentadas y a establecer sus nexos.

Es recomendable que el docente, en esta parte final de la clase, de manera creativa realice una motivación para la próxima clase teórica. Este as- 
pecto posibilita que los estudiantes se interesen por el tema que se va a estudiar posteriormente, que localicen fuentes de consulta relacionadas con este, es decir, que lleguen al momento en que se desarrolle esa clase con una información previa que les permita comprenderlo mejor y relacionarlo con lo ya conocido.

El proceder descrito favorece que los alumnos reconozcan los nexos que se establecen entre los diferentes temas que contempla el programa, lo que contribuye a su asimilación. Además, estimula su participación activa en el proceso de enseñanzaaprendizaje, pues durante la próxima clase teórica estos podrán, incluso, presentar interrogantes que les hayan surgido durante la autopreparación realizada.

Este tipo de clase, la conferencia, se utiliza con asiduidad desde el contexto de la universidad medieval (Valdaliso, 2010). En esta se concebía al docente como el poseedor de saberes enciclopédicos que debían ser transmitidos a los estudiantes. Estos los recibían como un todo único e inamovible, desde una posición pasiva que los situaba como simples receptores, es decir, era una enseñanza esencialmente memorística; a diferencia de la concepción expresada sobre el papel activo de los alumnos en el proceso.

En la actualidad, la clase teórica o conferencia sigue siendo de utilidad para el desarrollo del proceso de enseñanza-aprendizaje, especialmente en la enseñanza superior, que requiere abarcar amplios conocimientos en cada tema de un programa determinado. Estos posteriormente se aplican a través de actividades prácticas que realizan los estudiantes y se amplían mediante diferentes técnicas de estudio. Es necesario enfatizar que en una clase teórica el docente no agota todo el volumen de conocimientos de la rama del saber que se estudia, lo que además sería imposible por el cúmulo alcanzado en estos.

Es necesario apuntar que según los objetivos propuestos, el momento en el que se desarrolle esta forma de docencia, y las peculiaridades de la ma- teria, el docente puede planificar clases teóricas de diferentes tipos. Entre ellas pueden mencionarse las panorámicas y las especializadas o temáticas. Las panorámicas pueden ser útiles cuando se debe estudiar un amplio volumen de conocimientos, lo que puede hacer el profesor dada su preparación; subsiguientemente se podrá profundizar en otros temas contenidos en la generalización presentada, a través de clase teóricas especializadas o temáticas.

En este tipo de clase el docente reflexiona sobre aspectos cardinales para la comprensión de los fundamentos científico-técnicos más actualizados sobre un tema. Evidencia su capacidad y su experiencia pedagógica al dejar a los estudiantes orientados y motivados para continuar la búsqueda del conocimiento, lo que favorece el desarrollo de su independencia cognoscitiva.

Los elementos apuntados no deben indicar que en este tipo de clase solo se desarrollan contenidos cognoscitivos, en detrimento de los procedimentales y los actitudinales (Castellanos et al., 2002). Sin lugar a dudas, una clase teórica se concibe para desarrollar determinados conocimientos (los alumnos adquieren saberes sobre hechos, teorías, conceptos, entre otros aspectos), pero también favorece el desarrollo de habilidades intelectuales (Addine et al., 2004), se ha insistido en el papel activo de los alumnos, quienes deben: analizar, comparar, generalizar, sintetizar, entre otras habilidades.

En este sentido, cabe destacar que una clase teórica, al igual que la de otro tipo, no solo cumple una función instructiva, pues esta se relaciona de manera indisoluble con la educativa. Téngase presente que el desarrollo del proceso de enseñanzaaprendizaje exige la aplicación consecuente de diferentes principios que lo rigen (Addine et al., 2004), entre otros, el de la unidad de lo cognitivo y lo afectivo: se transmiten saberes y, mediante ellos, determinados valores.

En esta forma de docencia se contribuye al desarrollo axiológico de los estudiantes en la medida 
en que se promueven valores, actitudes, modos de actuación, mediante el contenido de enseñanzaaprendizaje. Piénsese, por ejemplo, que el profesor debe hacer un uso culto de la lengua materna, sirviendo de modelo a los alumnos, incidiendo, a partir de su ejemplo personal, en su competencia comunicativa (Niño, 2008), meta por lograr en el sistema educativo, de marcada importancia en su futura profesión y en lo personal.

Este tipo de clase, además, se caracteriza por su rigor científico al exponer los fundamentos teóricos sobre un tema de estudio. El docente muestra a los estudiantes las fuentes consultadas, presenta opiniones provenientes de los autores que se citan. El respeto que evidencia por las ideas ajenas repercute en una actitud ética en los alumnos, la que es esencial al desarrollar tareas investigativas y en su formación integral.

A manera de ejemplos se mencionan estos aspectos, que aportan al desarrollo de los contenidos actitudinales, en estrecho vínculo con los cognoscitivos y los procedimentales. Es necesario apuntar que, como se sabe, un tema tan complejo y necesario en el accionar pedagógico como el fortalecimiento de valores morales en los estudiantes no se agota en los mismos. Se requiere de una labor sistemática, aprovechando las potencialidades que ofrece el proceso, además de la influencia de la familia y la sociedad, pues no es tarea exclusiva de la institución universitaria, aunque a esta corresponde un trabajo intencionado, dirigido a la educación de la personalidad de los educandos.

\section{Recomendaciones metodológicas para el desarrollo de clases prácticas}

La forma de docencia denominada clase práctica es un tipo de clase que se dirige fundamentalmente al desarrollo de habilidades en los estudiantes. Mediante tareas docentes que elabora el profesor en relación con los objetivos, se propicia la aplicación, de manera independiente, de los conocimientos adquiridos. En la clase práctica los alumnos emplean métodos de trabajo específicos de la asignatura y de la futura profesión.

El adecuado desarrollo de este tipo de clase exige que se oriente con antelación una guía, mediante la cual se den las pautas esenciales a los estudiantes para su autopreparación. Dicha guía debe precisar el objetivo que se quiere lograr, de manera que los alumnos sepan lo que se espera de la actividad. Además, debe contener orientaciones metodológicas que expliquen el procedimiento que se va a seguir, actividades que permitan alcanzar el dominio de los aspectos teóricos necesarios para la clase, así como el desarrollo de las habilidades correspondientes, las fuentes bibliográficas por consultar y los indicadores por emplear en la evaluación.

En la introducción de la clase práctica el docente debe realizar una rememoración de los aspectos teóricos ya estudiados, y que son esenciales para el desarrollo de la misma, que se han de aplicar mediante tareas docentes. El profesor debe cerciorarse de la preparación que han logrado los estudiantes, a partir de la guía orientada; debe proceder a la retroalimentación que sea necesaria, en función del cumplimiento de los objetivos trazados, los que orientará de manera adecuada.

El desarrollo de la clase práctica debe iniciar con precisiones sobre la metodología que se seguirá en la realización de las tareas elaboradas. Los estudiantes deben saber la forma de trabajo que se empleará: individual o colectiva. Deben conocer cómo se realizará la evaluación de su desempeño mediante los indicadores establecidos, lo que tiene un importante valor educativo al contribuir a desarrollar su capacidad de autoevaluación, y, por consiguiente, el reconocimiento de logros, así como la asunción de dificultades, lo que debe influir en su mejora.

Es necesario que el docente, al preparar la clase práctica, a partir de los objetivos del programa y de las características de sus estudiantes, eleve de manera gradual la complejidad de las habilidades necesarias para solucionar las tareas que exigen la 
aplicación de los conocimientos adquiridos. De esta forma, puede ir logrando el tránsito de los alumnos de la dependencia a la independencia, dando al aprendizaje un carácter desarrollador (Castellanos et al., 2002), como se aspira.

Las tareas docentes para ejecutar en la clase práctica deben permitir a los alumnos establecer nexos con otros temas de la materia - relaciones intramateria- (Fiallo, 1997). Además, deben propiciar vínculos con los contenidos de otras asignaturas de la disciplina — relaciones intermateria - (Fia1lo, 1997). También, dichas tareas deben posibilitar el establecimiento de relaciones con otras disciplinas afines que conforman la malla curricular —interdisciplinariedad- (Ander-Egg, 1994; Torres, 2000).

En la actualidad, no caben dudas de lo que aporta al aprendizaje el establecimiento de nexos entre contenidos, métodos, habilidades (entre otras categorías) de diferentes materias o disciplinas. Este trabajo permite afianzar, consolidar, el contenido de enseñanza-aprendizaje que reciben los alumnos y ampliar la visión que van conformando sobre el mismo. El contenido no puede tratarse de manera segmentada, todo lo contrario, debe adquirir un enfoque holístico, integrador.

Para el logro de este propósito se requiere de un docente preparado científica y metodológicamente. Este debe dominar la materia que imparte, la disciplina a la que pertenece la misma, y, en general, la malla curricular para que pueda trabajar desde cada clase en función del logro de los objetivos planteados en el perfil de egreso de los estudiantes.

La preparación de la que se habla no solo debe entenderse desde el plano cognoscitivo, el que es esencial, por supuesto. Se trata de la actitud que debe tener el profesor para ampliar su perspectiva sobre los temas que imparte, para su autosuperación de manera permanente, la que comunicará a sus alumnos como un modo de actuación profesional.

En la clase práctica, la realización de las tareas docentes que se plantean a los estudiantes debe per- mitir el desarrollo de sus habilidades para el trabajo independiente (ya sea este individual o colectivo, en dependencia de lo planificado y orientado). Mientras los estudiantes realizan las tareas orientadas, el docente debe seguir su desempeño; para ello debe organizar el aula de manera tal que pueda pasar por los puestos de trabajo.

Este proceder es muy importante pues le permite estar en contacto con los alumnos, ejercer en ellos su influencia educativa, establecer una adecuada comunicación con los mismos. Además, de esa forma podrá constatar su preparación, el desarrollo de sus habilidades, el cumplimiento de las orientaciones dadas, y, en consecuencia, ofrecer las indicaciones que se requieran para favorecer el cumplimiento de los objetivos propuestos.

En la parte final del desarrollo de la clase práctica el docente debe realizar la evaluación del desempeño de los estudiantes, según los indicadores establecidos. Es recomendable emplear la autoevaluación y la coevaluación (Sanmartí, 2007), esenciales en la formación de los educandos, los que deben llegar a reconocer potencialidades y debilidades propias y en sus compañeros.

Es necesario preparar a los estudiantes, de manera paulatina, a partir del modelo que transmita el docente, para que con objetividad valoren los logros alcanzados, en su trabajo y en el de sus condiscípulos, a sabiendas de que siempre pueden superarse. Además, deben educarse para que sean capaces de reconocer deficiencias, siempre con el ánimo que contribuir a su mejora. El docente debe realizar la heteroevaluación (Sanmartí, 2007) al ofrecer sus criterios sobre lo logrado y señalar las medidas necesarias para realizar el seguimiento al aprendizaje de los alumnos, de manera diferenciada.

Las conclusiones de la clase práctica deben ser utilizadas para realizar generalizaciones de carácter teórico y metodológico, a partir del objetivo establecido. El docente puede precisar los fundamentos teóricos aplicados en las tareas desarrolladas, en dependencia de los resultados obtenidos. De igual 
modo, debe valorar el cumplimiento de la metodología planificada, enfatizando en el desarrollo de las habilidades propuestas. En esta parte de la clase puede asignar otras actividades que se requieran para estimular el aprendizaje de los alumnos.

Las consideraciones expresadas sobre la clase en la enseñanza superior, avaladas por la teoría existente (véanse los autores citados), la experiencia de los autores y la utilización de los métodos de investigación señalados, permiten reiterar que constituye la forma organizativa esencial en el proceso de enseñanza-aprendizaje. Lo aseverado no indica que se desconozca que todas las formas organizativas son importantes; su interrelación contribuye al desarrollo adecuado del proceso. Es pertinente señalar que la clase en sí tiene la peculiaridad de permitir que se manifiesten en ella todos los componentes de dicho proceso, que se ejerza el accionar instructivo y educativo del docente, en comunicación estrecha con el alumnado, lo que incide en su formación integral.

\section{Conclusiones}

El proceso de enseñanza-aprendizaje en el nivel superior tiene variadas formas organizativas, que resultan esenciales para el desarrollo de la malla curricular de la carrera, de las competencias necesarias para la futura profesión y el logro del perfil de egreso, que guardan estrecha relación con la clase.

La clase constituye la forma fundamental para la organización del proceso de enseñanza-aprendizaje universitario, donde este se manifiesta con toda su riqueza; en ella confluyen los diferentes procesos que en este nivel se desarrollan, contribuyendo a la formación integral de los estudiantes, meta suprema del sistema educativo.

La clase en la enseñanza superior puede tener carácter teórico o práctico, de ahí sus características peculiares, pero en sentido general se dirige a la adquisición de conocimientos, y al desarrollo de habilidades y valores.

Las recomendaciones metodológicas elaboradas para el desarrollo de clases teóricas y prácticas constituyen sugerencias para su mejora, preocupación constante de la comunidad educativa, consciente de sus potencialidades para contribuir a la educación de la personalidad de los estudiantes.

\section{Referencias}

Addine Fernández, F. et al. (2004). Didáctica: teoría y práctica. La Habana: Editorial Pueblo y Educación.

Aguilar, J. (2003). Historia de la Sociedad de la Información. Hacia la sociedad del conocimiento en revolución tecnológica. Alicante: Universidad de Alicante.

Álvarez de Zayas, C. (I999). La escuela en la vida. Didáctica. La Habana: Editorial Pueblo y Educación.

Ander-Egg, E. (1994). Interdisciplinariedad en educación. Buenos Aires: Editorial Magisterio del Río de la Plata.

Araque Jaramillo, W. (2012). La vinculación con la colectividad y la articulación universidad-sociedad. Ecuador: Área de Gestión y Coordinador del Observatorio de la PyME.

Área, M. (2002). Los medios, los profesores y el currículo. Barcelona: Sendai Ediciones. 
Argudín, Y. (2000). La educación superior para el siglo XXI. Didáctica, 36, 16-25. Universidad Iberoamericana Santa Fe.

Bermúdez Serguera, R. y Rodríguez Rebustillo, M. (1996). Teoría y metodología del aprendizaje. La Habana: Editorial Pueblo y Educación.

Borgobello, A., Peralta, N. y Roselli, N. (2010). El estilo docente universitario en relación al tipo de clase y a la disciplina enseñada.Liberabit,16 (1),7-16.Recuperado de http://www.scielo.org.pe/scielo.php\%3Fpid\%3DS1729$48272010000100002 \% 26$ script\%3Dsci arttext/

Bravo, J. L. (2003). Los medios tradicionales de enseñanza: uso de la pizarra y los medios relacionados. Madrid: ICE de la Universidad Politécnica.

Castellanos, D. et al. (2002). Aprender y enseñar en la escuela. La Habana: Editorial Pueblo y Educación.

Cabero, J. (2001). Tecnología educativa, diseño y utilización de medios para la enseñanza. Barcelona: Paidós.

Didriksson, A. (2003). La universidad del futuro. Un estudio sobre las relaciones entre la educación superior, la ciencia y la tecnología en Estados Unidos, Japón, Suecia y México. México: CISE-UNAM.

Escudero Muñoz, J. M. (2006). El espacio europeo de educación superior. ¿Será la hora de la renovación pedagógica de la universidad? Murcia: ICE, Universidad de Murcia.

Fiallo, J. (1997). La relación intermateria. La Habana: Editorial Pueblo y Educación.

Fuentes González, H. (2009). Pedagogía y didáctica de la educación superior. Santiago de Cuba: Universidad de Oriente, Centro de Estudio de Educación Superior Manuel F. Gran.

Frade Rubio, L. (2014). La evaluación basada en competencias. México, D.F.: Calidad Educativa Consultores.

González, A. (1990). Cómo propiciar la creatividad. La Habana: Editorial Ciencias Sociales.

Guzmán Cedeño, C. (2014). El proceso extensionista universitario como vía para la pertinencia en la formación del futuro profesional. ESPAMCIENCIA, 5 (1), 17-24.

Hernández Infante, R. C. e Infante Miranda, M. E. (2011). La relación del estudiante con la identidad: un acercamiento a través de la literatura local. Ciencias Holguín [Revista Electrónica], XVII (2), 125-140. Recuperado de http://www.ciencias.holguin.cu/

Mestre, U. et al. (2008). Fundamentos didácticos para un aprendizaje participativo. Bogotá: Universidad Libre.

Ministerio de Educación Superior (1991). Reglamento del trabajo docente metodológico en la Educación Superior. La Habana: MES.

Ministerio de Educación Superior (2006). La Nueva Universidad Cubana y su contribución a la universalización del conocimiento. La Habana: Editorial Félix Varela. 
ISSN $0123-1294$ | e-ISSN 2027-5358 | Educ.Educ. Vol. 20. No.1 | Enero-Abril de 2017 | pp. 27-40.

Universidad de La Sabana | Facultad de Educación

Ministerio de Educación Superior (2007). Reglamento docente metodológico. Resolución 210-2007. La Habana: MES.

Niño Rojas, V. (2008). Competencias en la comunicación. Hacia las prácticas del discurso. Bogotá: ECOE Ediciones.

Ontoria, A. et al. (2006). Aprender con mapas mentales: una estrategia para pensar y estudiar. Madrid: Narcea.

Rué, J. (2007). Enseñar en la universidad. El EEES como reto para la educación superior. Madrid: Narcea.

Sanmartí, N. (2007). 10 ideas clave. Evaluar para aprender. Barcelona: Graó.

Tobón, S. (2010). Formación integraly competencias (3 ed.). Bogotá: Ediciones ECOE.

Torres, J. (2000). Globalización e interdisciplinariedad: el currículo integrado. Madrid: Morata.

Tünnermann, C. (2006). La educación superior en el umbral del siglo XXI. Caracas: Cresal, Unesco.

Universidad del País Vasco (2006). Normativa sobre tipos de docencia y dimensionamiento de grupos en el marco de créditos europeos. Presentación para Consejo de Gobierno. Recuperado de http://www.ehu.eus/biofisica/juanma/mbb/docencia.htm/

Valdaliso, C. (2010). La vida de los estudiantes en la Edad Media. Barcelona: Historia de National Geographic, 72.

Valdés Veloz, H. (2013). Los principios y las funciones didácticas. Chile: Academia Nacional Iquique.

Ventosa, V. J. (2004). Métodos activos y técnicas de participación para educadores y formadores. Madrid: CCS.

Zabalza Beraza, M. A. (2004). Guía para la planificación didáctica de la docencia universitaria en el marco del EEES. Santiago de Compostela: Universidad de Santiago de Compostela. Recuperado de http://www.udc. es/grupos/apumefyr/docs/guiadeguias.pdf /

Zilberstein Toruncha, J. y Silvestre Oramas, M. (2000). ¿Cómo hacer más eficiente el aprendizaje? México: Ediciones CEIDE. 